\title{
MAXIMAL ORDERS IN RATIONAL CYCLIC ALGEBRAS OF COMPOSITE DEGREE*
}

\author{
BY \\ SAM PERLIS
}

Introduction. A maximal order $M$ of a normal division algebra $D$ over the rational number field may be imbedded $\dagger$ in a simple fashion in a maximal order of any normal simple algebra similar to $D$. When the normal simple algebra has degree greater than two, its class number is unity, $\ddagger$ and it can then be shown that all maximal orders of the algebra are obtainable from any one by an inner automorphism of the algebra. Thus it is sufficient to determine a single $M$ of each $D$ in order to determine all maximal orders of all normal simple algebras of degree greater than two over the rational number field. This determination was made by Hull§ for the case in which the degree $n$ of $D$ is any odd prime, using methods similar to those of Albert $\|$ for the case $n=2$. The methods and results of Hull are extended here to the case in which $n=\pi^{e}$ where $\pi$ is any odd prime, and also to the case $n=2^{e}>2$ provided that $D$ has odd discriminant and has the real number field as splitting field.

More specifically, it will be shown with the aid of the class field theory that each algebra $D$ considered has a suitably normalized cyclic generation, and a maximal order of $D$ will be expressed in terms of a finite number of quantities related to this generation. There are two chief points of difference between the present case and that of prime degree. The quantity $\sigma$ in the normalized generation $(Z, S, \sigma)$ is no longer the product of the primes ramified in $D$, but the product of certain powers of these primes. The exponents on these powers reduce to unity in the case of prime degree. The explicit basis given for the maximal order is similar to that for prime degree

* Presented to the Society, April 9, 1938; received by the editors October 13, 1938. While preparing this paper the author had the privilege of discussing some of its details with Professor A. A. Albert, and is grateful for this guidance and stimulus.

$\dagger$ For the concepts and results on the arithmetic of algebras see M. Deuring, Algebren,Ergebnisse der Mathematik und ihrer Grenzgebiete, vol. 4, no. 1.

$\ddagger$ M. Eichler, Bestimmung der Idealklassenzahl in gewissen normalen einfachen Algebren, Journal f:ir die reine und angewandte Mathematik, vol. 176 (1936), pp. 192-202.

$\S$ Ralph Hull, Maximal orders in rational cyclic algebras of odd prime degree, these Transactions, vol. 38 (1935), pp. 514-530. For the case $n=2$ see Hull's paper in the same journal, vol. 40 (1936), pp. 1-11. Reference to the first of these papers will be made by the letter $\mathrm{H}$.

II A. A. Albert, Integral domains of rational generalized quaternion algebras, Bulletin of the American Mathematical Society, vol. 40 (1934), pp. 164-176. 
except for the appearance of certain rational integral denominators which, again, reduce to unity in the case $n=\pi$.

For algebras of arbitrary degree, the determination may be reduced to the prime-power case if one can express maximal orders in a direct product of two normal division algebras of relatively prime degrees in terms of maximal orders in the two factors. A partial discussion of this direct product theory is given in the final section. The product of two orders, one in each factor, is an order in the direct product of the algebras, and it is shown that this order is maximal if and only if the discriminants of the two algebras are relatively prime. This result holds if any normal simple algebras are used instead of normal division algebras.

1. Cyclic generations and related concepts. A normal division algebra $D$ of degree $n$ over $R$ is a cyclic algebra

$$
D=(Z, S, \gamma)
$$

and has a basis

$$
u^{i-1} z_{j}, \quad i, j=1, \cdots, n,
$$

where $\left(z_{1}, \cdots, z_{n}\right)$ is a basis of the cyclic field $Z$ over $R$ with generating automorphism $S$, and

$$
u^{n}=\gamma, \quad z u=u z^{S}
$$

for every $z$ of $Z$.

Since $(Z, S, \gamma)=\left(Z, S, \gamma \rho^{n}\right)$ for any rational number $\rho \neq 0$, it follows that the quantity $\gamma$ of $R$ may be assumed with no loss of generality to be a rational integer. If we choose for the basis $\left(z_{1}, \cdots, z_{n}\right)$ a minimal basis of $Z$, then the set of all linear combinations of the $n^{2}$ quantities (2) with coefficients rational integers is an order of $D$. This order is uniquely determined by the cyclic generation (1) of $D$ and is called the order $I$ in $D$ associated with this generation. Every order of $D$, in particular an order $I$, is contained in a maximal order* of $D$. We shall obtain an infinite number of normalized cyclic generations of $D$ and for each of the corresponding orders $I$ we shall obtain $n$ distinct maximal orders containing $I$.

A complete set of invariants of $D$ under change of cyclic generation has been obtained by Hasse $\dagger$ in terms of the norm residue symbol

$$
(\gamma, Z \mid q)=\left(\frac{\gamma, Z}{q}\right)=S^{\nu} q
$$

* Deuring, op. cit., p. 70.

$\dagger \mathrm{H}$. Hasse, Theory of cyclic algebras over an algebraic number field, these Transactions, vol. 34 (1932), pp. 171-214. 
which is defined for every prime spot $q$ of $R$. We shall adopt the convention that the integer $\nu_{q}$ is one of $0,1, \cdots, n-1$. For any cyclic algebra $D_{1}=\left(Z_{1}, S_{1}, \gamma_{1}\right)$ of degree $n$ over $R$, we have $\left(\gamma_{1}, Z_{1} \mid q\right)=S_{1^{\nu_{1} q}}$, and Hasse has shown that $D_{1}$ is equivalent to $D$ if and only if $\nu_{1 q}=\nu_{q}$ for every $q$. Hence the $\nu_{q}$ and the degree $n$ form a complete set of invariants of $D$.

It is known that the norm residue symbol is the identity automorphism, that is, $\nu_{q}=0$, for all but a finite number of prime spots $q=q_{1}, \cdots, q_{s}$. These are precisely the prime spots for which the $q$-adic extension $D_{q}=D \times R_{q}$ is not total matric, and also are characterized as the prime factors of the discriminant of $D$. These prime spots $q_{1}, \cdots, q_{s}$ are called the ramification spots of $D$, and a cyclic algebra has at least two ramification spots unless it is total matric. The invariants $\nu_{q}$ satisfy the relations

$$
\sum_{q} \nu_{q} \equiv 0(\bmod n), \quad 2 \nu_{q_{\infty}} \equiv 0(\bmod n)
$$

where $q_{\infty}$ is the infinite prime spot of $R$, and these are the only relations between the invariants of an arbitrary cyclic algebra over $R$. However, a necessary and sufficient condition that a cyclic algebra $D$ of prime-power degree $n=\pi^{e}$ over $R$ be a division algebra is that at least one of its $q$-adic extensions be a division algebra, and this is equivalent to the condition that the corresponding invariant $\nu_{q}$ be prime to $n$. Both of these equivalent conditions follow readily from theorems* that (1) the $q$-index of $D=(Z, S, \gamma)$ over $R$ is the order of the automorphism $S^{\nu} q$ and thus is

$$
n_{q}=n /\left(n, v_{q}\right)
$$

and (2) the index of the cyclic algebra $D$ is the least common multiple of all of its $q$-indices $n_{q}$.

Until $\$ 5$ it will always be assumed that the normal division algebra $D$ has prime-power degree $n=\pi^{e}>2$ over $R$ so that there exists a $\nu_{q}$ which is prime to $n$. From (5) one obtains $\nu_{q_{\infty}}=0$ if $n$ is odd (so that in this case $q_{\infty}$ cannot be a ramification spot $\left.q_{i}\right)$, and $\nu_{q_{\infty}} \equiv 0(\bmod 2)$ if $n=2^{e}>2$. In any case, $\nu_{q_{\infty}}$ is not prime to $n$. Conditions (5) also imply that $s \geqq 2$ and that there must be at least two prime spots for which the corresponding invariants are prime to $n$. Hence we may hereafter let $q_{1}$ designate a ramification spot such that $\left(\nu_{q_{1}}, n\right)=1$ and $q_{1} \neq \pi$.

2. Normalized cyclic generations. Three lemmas will now be obtained for use in the proof of Theorem 1 which provides cyclic generations of an especially simple type for the algebra $D$. The first lemma defines a collection of fields from which the cyclic generation fields of $D$ will be selected.

\footnotetext{
* Hasse, ibid., Theorem 5, p. 179, and (17.7), p. 203.
} 
Lemma 1. For any prime $p \equiv 1(\bmod 2 n)$ let $H_{p}$ be the ideal group in $R$ consisting of all principal ideals $(r)$ where $r$ is a rational number prime to $p$ and is an $n$-ic residue modulo $p$. Then the class field $Z_{p}$ corresponding to $H_{p}$ is cyclic of degree $n$ over $R$ and has conductor $p$.

If we let $G_{p}$ be the group of all ( $\left.r\right)$ with $r$ prime to $p$ and let $g$ be a primitive root of $p$, we shall verify the decomposition

$$
G_{p}=H_{p}+H_{p} g+\cdots+H_{p} g^{n-1} .
$$

When $p \equiv 1(\bmod 2 n)$, a quantity $\pm g^{i}$ is an $n$-ic residue modulo $p$ if and only if $i$ is a multiple of $n$, whence it follows that the cosets $H_{p} g^{i}$ are distinct. For any $(r)$ in $G_{p}$ we have $r=a b^{-1}, a$ and $b$ integers prime to $p, a=g^{e}+x p$, $b=g^{f}+y p$ with integers $x$ and $y$. Then

$$
r=\frac{g^{f}+x_{1} p}{g^{f}+y p} g^{e-f}=r_{1} g^{e-f}=\frac{g^{e}+x p}{g^{e}+y_{1} p} g^{e-f} .
$$

If $f \geqq e$, the number $x_{1}$ is an integer, and we have $r_{1} \equiv 1(\bmod p)$. Otherwise $y_{1}$ is integral and again we have the same congruence, so that $\left(r_{1}\right)$ is in $H_{p}$ and $(r)$ is in $H_{p} g^{e-f}$, which is one of the cosets displayed. This verifies the decomposition above. The prime $p$ is a generating modulus of the ideal group $H_{p}$ so that the conductor of $H_{p}$, which is the g.c.d. of all the generating moduli, is either $p$ or 1 . Then clearly the conductor of $H_{p}$, and hence of $Z_{p}$, is $p$; and since $G_{p} / H_{p}$ is cyclic of order $n$, the field $Z_{p}$ is cyclic of degree $n$ over $R$.

Since the next two lemmas depend on the notations of Theorem 1, the latter result will be stated now but not proved until the lemmas have been obtained.

THEOREM 1. Let $D$ be a normal division algebra of prime-power degree $n=\pi^{e}$ over the rational number field $R$, and let $q_{1}, \cdots, q_{s}$ be the finite ramification spots of $D$ and $n_{i}$ the $q_{i}$-index of $D,(i=1, \cdots, s)$. Then, if $\pi$ is odd, there are infinitely many cyclic fields $Z$ of degree $n$ over $R$ such that

(a) $D=(Z, S, \sigma), \sigma=\prod_{i=1}^{s} q_{i}{ }^{n / n_{i}}$;

(b) $Z$ has conductor a prime $p$ such that $p \equiv 1(\bmod n),(p, \sigma)=1$;

(c) $q_{1}, \cdots, q_{s}$ generate prime ideals $\left(q_{i}\right)$ in $Z$;

(d) $\sigma$ is an $n$-ic residue modulo $p$. If $n=2^{e}>2$ the same results hold provided that $D$ is unramified at the prime spot 2 and at the infinite prime spot $q_{\infty}$.

Let $\nu_{\infty}$ and $\nu_{1}, \cdots, \nu_{s}$ be the invariants corresponding to $q_{\infty}$ and the $q_{i}$. As we have already seen, our hypotheses imply that $\nu_{\infty}=0$. By (6) we have $n_{i}=n /\left(n, \nu_{i}\right)$, and the congruences 


$$
-\nu_{1} n n_{i}^{-1} x_{i} \equiv \nu_{i}(\bmod n) \text {, }
$$$$
i=2, \cdots, s,
$$

have solutions $x_{i}$ since $\left(n, \nu_{1}\right)=1$ and $\left(n, \nu_{1} n n_{i}^{-1}\right)=\left(n, n n_{i}^{-1}\right)=n n_{i}^{-1}=\left(n, \nu_{i}\right)$. Note that the $x_{i}$ are prime to $n$. Let $\zeta$ be a primitive $n$th root of unity; let

$$
\begin{aligned}
& \alpha_{i}=\left(q_{1}^{x_{i}} q_{i}\right)^{n / n_{i}}, \\
& i=2, \cdots, s \text {, } \\
& F=R(\zeta), \quad K=F\left(\alpha_{2}{ }^{1 / n}, \cdots, \alpha_{s}^{1 / n}\right) .
\end{aligned}
$$

Lemma 2. The field $K_{1}=K\left(q_{1}^{1 / \pi}\right)$ has degree $\pi$ over $K$.

Consider an equation

$$
q_{1}^{c_{1}}\left(q_{1}^{x_{2}} q_{2}\right)^{c_{2}} \cdots\left(q_{1}^{x_{s}} q_{8}\right)^{c_{s}}=a^{n}, \quad a \text { in } F,
$$

where the $c_{i}$ are integers to be determined, and suppose that $\pi$ is not one of the $q_{i}$. Then all the $q_{i}$ are unramified in $F$ since the discriminant of $F$ is a power* of $\pi$. Hence the prime ideal factorization of the quantities in (11) shows that $c_{1}+c_{2} x_{2}+\cdots+c_{s} x_{s}$ and $c_{2}, \cdots, c_{s}$ are all divisible by $n$, and therefore $c_{1}$ is divisible by $n$. Thus (11) holds only when the exponents $c_{i}$ are all multiples of $n$, a property which implies $\dagger$ that the composite of the fields $F\left(q_{1}^{1 / n}\right)$ and $F\left(\left[q_{1}^{x_{i}} q_{i}\right]^{1 / n}\right)$ for $i=2, \cdots, s$ is their direct product. These $s$ fields have subfields $F\left(q_{1}^{1 / \pi}\right)$ and $F\left(\alpha_{i}^{1 / n}\right)$ for $i=2, \cdots, s$, respectively, and the composite of these subfields must be their direct product. Then the degree of $K_{1}$ over $K$ is the degree of $F\left(q_{1}^{1 / \pi}\right)$ over $F$, and this is either $\ddagger \pi$ or 1 . If the degree were 1 , then $F$ would contain $q_{1}^{1 / \pi}, q_{1}$ would be the $\pi$ th power of an ideal in $F$, whereas $q_{1}$ is prime to $\pi$ and hence unramified in $F$. We have proved the lemma for the case in which $\pi$ is not one of the $q_{i}$.

In case $\pi$ is one of the $q_{i}$, we have assumed $\pi>2$ and may take $\pi=q_{2}$. Consider an equation of the form (11) with the factor $q_{1}{ }^{c_{1}}$ deleted, and obtain $c_{2} x_{2}+\cdots+c_{s} x_{s} \equiv c_{3} \equiv \cdots \equiv c_{s} \equiv 0(\bmod n)$ since $q_{1}$ and $q_{3}, \cdots, q_{s}$ are unramified in $F$. Thus $c_{2} x_{2}$ is divisible by $n, x_{2}$ is prime to $n$, and $c_{2} \equiv 0(\bmod n)$. As in the previous paragraph, the composite $K_{0}$ of the fields $F\left(\left[q_{1}{ }^{x_{i}} q_{i}\right]^{1 / n}\right)$ for $i=2, \cdots, s$ is then their direct product and (by Bericht II, p. 43) any cyclic subfield of $K_{0}$ has the form

$$
F\left(\left[\left(q_{1}^{x_{2}} q_{2}\right)^{d_{2}} \cdots\left(q_{1}^{x_{s}} q_{s}\right)^{d_{s}}\right]^{1 / n}\right),
$$

$d_{i}$ integers.

If $F\left(q_{1}^{1 / \pi}\right)$ is contained in $K_{0}$, it must have the form (12) so that

* R. Fricke, Lehrbuch der Algebra, 1928, vol. 3, p. 195.

$\dagger \mathrm{H}$. Hasse, Bericht über neuere Untersuchungen und Probleme aus der Theorie der algebraischen Zahlkörper, Teil II, Jahresbericht der deutschen Mathematiker-Vereinigung, supplementary vol. 6 (1930), p. 43. Parts I and Ia of this article appeared in the Jahresbericht, vols. 35 and 36 . These papers will be designated here as Bericht I, Ia, and II.

$\ddagger$ Bericht II, p. 42, Theorem I.

§ Bericht II, p. 42, Theorem II. 


$$
q_{1}{ }^{n / x}=c^{n} q_{1}{ }^{\left(x_{2} d_{2}+\cdots+x_{s} d_{s}\right) x} \pi^{d_{2} x} \cdot q_{3}^{d_{3} x} \cdots q_{s}^{d_{s} x}
$$

with $c$ in $F$. By considering prime ideal factorizations of the quantities in this equation, we find that $d_{3} x, \cdots, d_{s} x$ are divisible by $n, x_{2} d_{2} x+\cdots+x_{8} d_{s} x$ $\equiv x_{2} d_{2} x \equiv n / \pi(\bmod n), x_{2}$ is prime to $n, d_{2} x \equiv x_{0} n / \pi(\bmod n)$. The equation above then takes the form

$$
q_{1}^{n / \pi}=c_{0}{ }^{n} q_{1}^{n / \pi} \pi^{x_{0} n / \pi}, \quad c_{0}^{-n}=\pi^{x_{0} n / \pi} .
$$

Since $x_{0}$ is prime to $n$ we easily obtain $\pi^{n / \pi}=c_{01}^{n}$ with $c_{01}$ in $F$ and thus have $\pi^{\prime / \pi}$ in $F$. Then $F$ must contain the non-normal subfield $R\left(\pi^{1 / \pi}\right)$ whereas $F$ is cyclic and all of its subfields are normal over $R$. We have shown that $F\left(q_{1}^{1 / \pi}\right)$ is not contained in $K_{0}$. Then it is not contained in the subfield $K$ of $K_{0}$ and the lemma is proved. $\dagger$

LEMma 3. There are infinitely many rational primes $p$ such that $p \equiv 1$ $(\bmod 2 n),(p, \sigma)=1$, and

(e) $\alpha_{2}, \cdots, \alpha_{s}$ are $n$-ic residues modulo $p$;

(f) $q_{1}{ }^{t}$ is an $n$-ic non-residue modulo $p$ for $t=1, \cdots, n-1$.

The field $K_{1}$ of Lemma 2 is cyclic of degree greater than 1 over $K$ and is class field to an ideal group $H_{1}$ in $K$. In any ideal class different from the identity class $H_{1}$, we may select an infinite number of prime ideals $P$ which are of degree one, prime to $\sigma$, and prime to the different of $K$ over $R$. An infinite number of rational primes $p=N_{K \mid R}(P)$ is thus defined. Every such $p$ is prime to $\sigma$; and since the prime ideal factors of $p$ in $F$ must have degree one, it follows that $p \equiv 1(\bmod n)$. Then $p \equiv 1(\bmod 2 n)$ if $n$ is odd.

When $n=2^{e}$ we shall make the following additional restrictions in the choice of the ideals $P$. Let $F_{2}$ be the root field over $R$ of the equation $x^{2 n}=1$ so that $F_{2}$ has degree two over $F$. The field $K$ cannot contain $F_{2}$ since then $F_{2}$ would have the form (12) which leads to a contradiction. Hence the composite $\left(K, F_{2}\right)$ has degree two over $K$ and is the class field corresponding to an ideal group $H_{2}$ in $K$. We wish to choose ideals $P$ lying outside of $H_{1}$ as before but also lying in $H_{2}$. Let these ideal groups have a common generating modulus. Then $H_{1}$ and $H_{2}$ are collections of ray classes, and we must verify that the ray classes comprising $H_{2}$ do not all lie among those comprising $H_{1}$. This fact is clearly true since otherwise $\left(K, F_{2}\right)=K_{1}, K\left(\zeta^{1 / 2}\right)=K\left(q_{1}^{1 / 2}\right)$, which is impossible. Thus there is a ray class $C$ in $H_{2}$ but not in $H_{1}$, and $C$ contains infinitely many prime ideals with the properties of the previous paragraph.

† Since $\left(q_{1}^{x_{2}} \pi\right)^{1 / n_{2}}$ is in $K$, this field contains $q_{1}^{1 / \pi}$ if and only if it contains $\pi^{1 / \pi}$. Then we see that Lemma 2 is false without the hypothesis that $\pi \neq 2$ when $\pi$ is one of the $q_{i}$. For, if $\pi=2$, take $n \geqq 8$ and see that $F$, and hence $K$, contains a primitive eighth root $\zeta_{0}$ of unity and thus contains $\zeta_{0}-\zeta_{0}{ }^{3}=2^{1 / 2}$, so that $q_{1}^{1 / 2}$ is in $K$ and the lemma fails. 
The norms of these ideals are rational primes $p$ such that $p \equiv 1(\bmod 2 n)$ since they are unramified in $F_{2}$ and their prime ideal factors in $F_{2}$ have degree one.

The proofs of properties (e) and (f) are similar to corresponding proofs in $\mathrm{H}$ and will be omitted here.* To prove Theorem 1 , let $p$ be any prime of Lemma 3 and let $Z$ be the corresponding field $Z_{p}$ of Lemma 1 . Then property (b) of the theorem holds. Property (f) of the last lemma is equivalent to the statement that $q_{1}$ is a prime ideal in $Z$, and property (e) implies that the $\alpha_{i}$ are in the ideal group $H_{p}$ corresponding to $Z$. Expressed in terms of Artin symbols these facts yield

$$
\left(Z / \alpha_{i}\right)=I, \quad\left(Z / q_{1}\right)^{x_{i n / n_{i}}}=\left(Z / q_{i}\right)^{-n / n_{i}} .
$$

Since $x_{i}$ is prime to $n$ and the automorphism $\left(Z / q_{1}\right)$ has order $n$, it follows that $\left(Z / q_{1}\right)^{x_{i n} / n_{i}}$ has order $n_{i}$. A simple computation shows that $\left(Z / q_{i}\right)$ has order $n$, which is equivalent to (c). Applying (e) together with (8) and (5), we are led to (d).

The Artin symbol $A=\left(Z / q_{1}\right)$ is a generating automorphism of $Z$ over $R$, and the equation $S^{\nu_{1}}=A^{-1}$ defines another generating automorphism $S$. Then $(Z, S, \sigma)$ is a cyclic algebra of degree $n$. A computation following the pattern in $\mathrm{H}$ shows that $D$ and $(Z, S, \sigma)$ have the same invariants, yielding (a) and completing the proof of the theorem.

3 . Some properties of $Z$. Since $Z$ is cyclic over $R$ with conductor $p$, it is a subfield $\dagger$ of the cyclotomic field $R(\xi)$, where $\xi$ is a primitive $p$ th root of unity. The field $R(\xi)$ is cyclic over $R$ so that $Z$ is its unique subfield of degree $n$, and $Z$ is thus uniquely determined by its degree $n$, its prime conductor $p$, and the property of being an abelian field over $R$. Write $p=1+h n$, and let $g$ be a primitive root of $p$. Then a normal basis of $Z$ is given by $\ddagger$

with

$$
\eta_{0}, \eta_{1}, \cdots, \eta_{n-1}
$$

$$
\eta_{i}=\xi_{i}+\xi_{i+n}+\cdots+\xi_{i+(h-1)}, \xi_{k}=\xi^{u^{k}},
$$

* We may observe that Lemma 3 is actually false without the assumption $\pi \neq 2$ when $\pi$ is one of the $q_{i}$. For, without this assumption we may have $n=2^{e}, K \geqq F\left(q_{1}^{1 / 2}\right)=K_{0}$, and $H \leqq H_{0}$, where $H$ and $H_{0}$, respectively, are the ideal groups in $F$ corresponding to the class fields $K$ and $K_{0}$ over $F$. The condition $p \equiv 1(\bmod n)$ implies that any prime factor $P$ of $p$ in $F$ has degree 1 , and condition (e) implies that $P$ is in $H$ and hence in $H_{0}$. Then any prime factor $P_{0}$ of $P$ in $K_{0}$ has degree 1 ; hence the quantity $q_{1}^{1 / 2}$ of $K_{0}$ satisfies $q_{1}^{1 / 2} \equiv y\left(\bmod P_{0}\right)$ with $y$ in $R$. Then $q_{1}^{n / 2} \equiv y^{n}\left(\bmod P_{0}\right)$ so that we have $q_{1}{ }^{n / 2} \equiv y^{n}(\bmod p)$, a contradiction with (f).

The falsity of Lemma 3 can be seen to imply the falsity of the conclusions in Theorem 1. Thus the restrictive assumption in Theorem 1 is necessary.

$\dagger$ Bericht I, p. 39.

$\ddagger$ B. L. van der Waerden, Moderne Algebra, 1930, vol. 1, pp. $160 \mathrm{ff}$. 
for $i=0, \cdots, n-1$ and $k=0,1, \cdots, p-2$. Hence $Z=R\left(\eta_{i}\right)$ for any $i$, and a generating automorphism of $Z$ over $R$ is induced by

$U$ :

$$
\xi \longleftrightarrow \xi^{\circ} .
$$

Clearly, $U$ is a generating automorphism of the cyclic group [ $U$ ] of $R(\xi)$ over $R$, and $\left[U^{n}\right]$ is the group of $R(\xi)$ over $Z$.

The factorization of $p$ in $Z$ may now be obtained. Define

$$
\beta=\prod_{t=0}^{h-1}\left(1-\xi^{n t}\right) .
$$

Then $\beta$ is unaltered by $U^{n}$ and hence is in $Z$, and a direct computation shows that $N_{Z \mid R}(\beta)=p$. The principal ideal $P=(\beta)$ is thus a prime ideal of $Z$ and is a factor of $p$. But $p$ is completely ramified in the cyclotomic field $R(\xi)$ and hence in the subfield $Z$, so that $p=P^{n}$. This fact and Theorem (14) of $\S 8$, Bericht Ia, may be used to show that the discriminant of $Z$ over $R$ is $p^{n-1}$. We thus have

Theorem 2. Each field $Z$ of Theorem 1 (and $Z_{p}$ of Lemma 1) has discriminant $p^{n-1}$. The factorization of $p$ in $Z$ is

$$
p=P^{n}, \quad P=(\beta), \quad N_{Z \mid R}(\beta)=p,
$$

where $\beta$ is given by (15).

The quantity $\beta$ will be used in the next section when basal elements of maximal orders are defined.

4. Maximal orders in $D$. The algebra $D$ has the form

$$
D=Z+u Z+\cdots+u^{n-1} Z, \quad u^{n}=\sigma,
$$

and this generation of $D$ is associated with an order

$$
I=Z_{0}+u Z_{0}+\cdots+u^{n-1} Z_{0}
$$

where $Z_{0}$ is the maximal order of $Z$. We shall display $n$ distinct maximal orders in $D$ which contain $I$. These $n$ orders are defined in terms of $n$ rational integers $\lambda$ given in

Lемма 4. The simultaneous congruences

$$
\lambda^{n} \equiv \sigma(\bmod p), \quad \lambda \equiv 0(\bmod \sigma)
$$

have exactly $n$ solutions $\lambda$ which are incongruent modulo $p$.

Any solution of the second congruence has the form $\lambda_{0} \sigma$. If this is substituted in the first congruence, there results

$$
\lambda_{0}{ }^{n} \equiv \sigma \sigma_{1}^{n}(\bmod p)
$$


with $\sigma \sigma_{1} \equiv 1(\bmod p)$. There exists a solution of (18) if and only if* we have $\left(\sigma \sigma_{1}\right)^{(p-1) / g} \equiv 1(\bmod p)$ where $g=(p-1, n)$; then the exact number of incongruent solutions is $g$. In the present case $g=n$, and the first congruence in (17) has a solution, by Theorem 1 , so that $\sigma^{(p-1) / n} \equiv 1(\bmod p)$. Also, $\sigma_{1}{ }^{p-1} \equiv 1(\bmod p)$ so that

$$
\sigma^{(p-1) / n} \sigma_{1}^{p-1}=\left(\sigma \sigma_{1}^{n}\right)^{(p-1) / n} \equiv 1(\bmod p),
$$

and the lemma is proved.

We shall consider modules of the form

$$
M=Z_{0}+y \tau_{1}^{-1} Z_{0}+\cdots+y^{n-1} \tau_{n-1}^{-1} Z_{0}
$$

where

$$
y=(\lambda-u) \beta^{-1}
$$

with $\lambda$ satisfying (17), $\beta$ given by (15), and where the $\tau_{j}$ are rational integers such that

$$
\tau_{n-1} \text { divides } \sigma, \quad \tau_{i} \text { divides } \tau_{i+1}
$$

for $i=1, \cdots, n-2$. The $\tau_{j}$ will be chosen so that $M$ is a ring. First, for any $a_{0}$ in $Z_{0}$ we find by a simple computation that $a_{0} y=\left(a_{0}-a_{0}{ }^{S}\right) \lambda \beta^{-1}+y a_{0}{ }^{S}$. The ramification order of $p$ in $Z$ over $R$ is $n$ so that the inertial group of $p$ in $Z$ over $R$ is the complete galois group of $Z$ over $R$. Hence $a_{0} \equiv a_{0} s(\bmod \beta)$ and we have $a_{0} y=y a_{0}{ }^{S}+a_{1} \lambda$, $\left(a_{1}\right.$ in $\left.Z_{0}\right)$. A simple induction then yields

LemMa 5. For every $a_{0}$ in $Z_{0}$ and every integer $i>0$ we have

$$
a_{0} y^{i}=y^{i} a_{0}{ }^{i}+y^{i-1} a_{1} \lambda+\cdots+a_{i} \lambda^{i},
$$

$a_{j}$ in $Z_{0}$.

By means of an $n$-rowed matrix representation of $D$ it may be verified $\dagger$ that the characteristic function of $y$ is

$$
t^{n}-\lambda \delta_{1} t^{n-1}+\lambda^{2} \delta_{2} t^{n-2}-\cdots+(-\lambda)^{n-1} \delta_{n-1} t+(-1)^{n} \delta_{n}
$$

where $\delta_{n}$ is the rational integer $\delta_{n}=\left(\lambda^{n}-\sigma\right) p^{-1}$ and, for $i<n, \delta_{i}$ is the $i$ th elementary symmetric function of $\beta^{-1}$ and its conjugates. The $i$ th elementary symmetric function of the algebraic integer $p \beta^{-1}$ and its conjugates in $Z$ is $p^{i} \delta_{i}$ which must then be a rational integer. Since $p^{i} \delta_{i}$ is divisible by

$$
P^{i(n-1)}=P^{(i-1) n+n-i}=\left(p^{i-1}\right) P^{n-i},
$$

it follows that $p \delta_{i}$ is a rational integer divisible by $P^{n-i}$ and hence by $p$ when $i<n$. This proves that all of the coefficients of (22) are rational integers.

* L. E. Dickson, Introduction to the Theory of Numbers, 1931, p. 31, exercise 5.

$\dagger$ See H, p. 525. 
Observe that the coefficient $\delta_{n}$ in (22) has the property that $\delta_{n} \sigma^{-1}$ is an integer prime to $\sigma$. An induction based on (22) yields

Leмma 6. For $k=0,1, \cdots, n-2$ we have

$$
y^{n+k}=y^{n-1} a_{1}+y^{n-2} a_{2}+\cdots+a_{n}
$$

with rational integral coefficients $a_{j}$ such that

$$
\begin{aligned}
a_{j} & \equiv 0\left(\bmod \lambda^{k+j}\right), & j=1, \cdots, n-k-1, \\
a_{n-k} & \equiv 0(\bmod \sigma), & \left(a_{n-k} \sigma^{-1}, \sigma\right)=1,
\end{aligned}
$$

and, if $k>0$,

$$
a_{j} \equiv 0\left(\bmod \lambda^{k+j+1-n}\right), \quad j=n-k+1, \cdots, n .
$$

Thus every $a_{j}$ is divisible by $\sigma$.

The module $M=Z_{0}+\sum_{i=1}^{n-1} y^{i} \tau_{i}^{-1} Z_{0}$ of (19) contains the set $M Z_{0}$, that is, all sums of products $a a_{0}$ with $a$ in $M$ and $a_{0}$ in $Z_{0}$. By Lemma 5, (21), and the fact that $\lambda$ is divisible by $\sigma$, we see also that the sets $Z_{0} y^{i} \tau_{i}^{-1}$ are all contained in $M$ so that $Z_{0} y^{i} \tau_{i}^{-1} Z_{0} \leqq M, Z_{0} M \leqq M$. Thus $M$ is a ring if and only if we have

$$
y^{i} \tau_{i}^{-1} M \leqq M,
$$$$
i=1, \cdots, n-1 \text {. }
$$

This is equivalent to the condition

$$
y^{i} \tau_{i}^{-1} y^{j} \tau_{j}^{-1}=y^{i+j}\left(\tau_{i} \tau_{j}\right)^{-1} \text { in } M, \quad i, j=1, \cdots, n-1 .
$$

When $i+j<n$, the condition (24) holds if and only if $\tau_{i} \tau_{j}$ divides $\tau_{i+j}$. Otherwise $i+j=n+k,(k=0,1, \cdots, n-2)$, and, by Lemma $6,(24)$ holds if and only if $\tau_{i} \tau_{j}$ divides each quantity $a_{r} \tau_{n-r},(r=1, \cdots, n)$, where we define $\tau_{0}=1$. In particular, it is sufficient to have

$$
\begin{aligned}
\sigma^{k+r} \tau_{n-r} & \equiv 0\left(\bmod \tau_{i} \tau_{j}\right), & r=1, \cdots, n-k-1, \\
\sigma^{k+r+1-n} \tau_{n-r} & \equiv 0\left(\bmod \tau_{i} \tau_{j}\right), & r=n-k, \cdots, n .
\end{aligned}
$$

Since $\tau_{i} \tau_{j}$ divides $\sigma^{2}$, (25) holds when $k+r \geqq 2$. Otherwise $r=1, k=0$, and (25) becomes $\sigma \tau_{n-1} \equiv 0\left(\bmod \tau_{i} \tau_{j}\right)$ which by (21) is satisfied. In (26) we have $k+r \geqq n$, and see that the condition is not restrictive when $k+r>n$, $k+r+1-n \geqq 2$. We have proved

LEMma 7. Sufficient conditions that the module $M$ of (19) be a ring are given by the following congruences:

$$
\begin{aligned}
\tau_{i+j} & \equiv 0\left(\bmod \tau_{i} \tau_{j}\right), & & i+j<n, \\
\sigma \tau_{i+j-n} & \equiv 0\left(\bmod \tau_{i} \tau_{j}\right), & & i+j \geqq n .
\end{aligned}
$$

Let us now make the definition $\tau_{0}=1$, 


$$
\tau_{j}=\prod_{i=1}^{s} q_{i}{ }^{e_{i}}, \quad e_{i}=\left[\frac{j}{n_{i}}\right]
$$

for $j=1, \cdots, n-1$, and verify that this choice of the $\tau_{j}$ satisfies the conditions* of Lemma 7 . The quantity $\tau_{a} \tau_{b}$ is exactly divisible by $q_{i 0}=q_{i}{ }^{e+f}$, $e=\left[a / n_{i}\right], f=\left[b / n_{i}\right]$. If $a+b<n$, the quantity $\tau_{a+b}$ is exactly divisible by $q_{i}{ }^{o}, g=\left[(a+b) / n_{i}\right] \geqq e+f$, so that (27) holds. If $a+b \geqq n$, then $\tau_{a+b-n}$ has the exact factor $q_{i}{ }^{\text {, }}$

$$
g=\left[\frac{a+b-n}{n_{i}}\right]=\left[\frac{a+b}{n_{i}}\right]-\frac{n}{n_{i}} \geqq e+f-\frac{n}{n_{i}} .
$$

But $\sigma$ has the factor $q_{i}{ }^{n / n_{i}}, \sigma \tau_{a+b-n}$ has $q_{i}{ }^{o+n / n_{i}} \geqq q_{i}{ }^{e+f}$ as factor, so that (28) holds. We have proved that $M$ is a ring.

The ring $M$ is a linear set of finite order over the domain of all rational integers; it contains $Z_{0}$ and hence all rational integers; and it contains $u=\lambda-y \beta$ and hence a basis $u^{i-1} z_{j},(i, j=1, \cdots, n)$, of $D$ where the $z_{j}$ form any integral basis of $Z$. These properties imply that the quantities of $M$ are all integral and that $M$ is an order of $D$. This order is maximal in $D$ if and only if $\ddagger$ its discriminant is the discriminant $\S$

$$
\prod_{i=1}^{s} q_{i}^{n^{2}\left(n_{i}-1\right) / n_{i}}
$$

of the algebra $D$.

The sets $M$ and $I$ have respective bases $w$ and $v$ given by the vectors

$$
\begin{aligned}
w & =\left(z_{1}, \cdots, z_{n}, \tau_{1}^{-1} y z_{1}, \cdots, \tau_{1}^{-1} y z_{n}, \cdots, \tau_{n-1}^{-1} y^{n-1} z_{n}\right)=\left(w_{1}, \cdots, w_{n 2}\right), \\
v & =\left(z_{1}, \cdots, z_{n}, u z_{1}, \cdots, u z_{n}, \cdots, u^{n-1} z_{n}\right)=\left(v_{1}, \cdots, v_{n 2}\right),
\end{aligned}
$$

where the $z_{j}$ form an integral basis of $Z$. There is a nonsingular matrix $B$ with rational elements such that $w=v B$, and the discriminant of $M$ is then

$$
\Delta(w)=\left|T\left(w_{i} w_{j}\right)\right|=\Delta(v) \cdot|B|^{2} .
$$

Here $\Delta(v)$ is the discriminant $\left|T\left(v_{i} v_{j}\right)\right|$ of the basis $v$, and $\mid \Delta(v)=(\sigma p)^{n(n-1)}$. To compute $|B|^{2}$ we observe $\uparrow$ that when the matrix $B$ is expressed as an

* Note that this choice of the $\tau_{j}$ makes $\tau_{1}, \cdots, \tau_{n_{i-1}}$ prime to $q_{i}$. Hence $\tau_{1}=\cdots=\tau_{\pi-1}=1$.

$\dagger$ Deuring, op. cit., p. 71, Theorem 9.

‡ E. Artin, Zur Arithmetik hyperkomplexer Zahlen, Abhandlungen aus dem mathematischen Seminar der Hamburgischen Universität, vol. 5 (1928), p. 265.

$\S$ Reichhardt, Die Diskriminante einer normalen einfachen Algebra, Journal für die reine und angewandte Mathematik, vol. 173 (1935), pp. 31-34.

\| See H, p. 523.

१ Ibid., p. 526. 
$n$-rowed matrix whose elements are $n \times n$ matrices $B_{i j}$, then every $B_{i j}$ below the main diagonal is a zero matrix, $B_{11}$ is an identity matrix, and every matrix $B_{i j},(j>1)$, has determinant equal, except possibly for sign, to the norm

$$
\left[N\left(\tau_{j-1} \beta \beta^{S} \ldots \beta^{S^{j-2}}\right)\right]^{-1}=\left(\tau_{j-1}^{n} p^{j-1}\right)^{-1} .
$$

Then $|B|^{2}=\left|B_{11} B_{22} \cdots B_{n n}\right|^{2}$ has the value

$$
|B|^{2}=\left(\tau_{1} \cdots \tau_{n-1}\right)^{-2 n} p^{-n(n-1)}
$$

so that $\Delta(w)=\sigma^{n(n-1)}\left(\tau_{1} \cdots \tau_{n-1}\right)^{-2 n}$. But

$$
\tau_{1} \cdots \tau_{n-1}=\prod_{i=1}^{s} q_{i}^{\left[\left(n / n_{i}-1\right)+\left(n / n_{i}-2\right)+\cdots+1\right] n_{i}}=\prod q^{\left(n^{\left(n / n_{i}-1\right) n / 2}\right.}
$$

and $\Delta(w)=\prod q_{i}{ }^{{ }^{2}\left(n_{i}-1\right) / n_{i}}$ which is the formula (30) for the discriminant of $D$. Thus $M$ is a maximal order of $D$.

THEOREM 3. Let $D$ be an algebra of Theorem 1 with normalized cyclic generation $(Z, S, \sigma)$ as described in that theorem, $D=Z+u Z+\cdots+u^{n-1} Z, u^{n}=\sigma$. Then $n$ distinct maximal orders in $D$ are given by the modules

$$
M(\lambda)=Z_{0}+y \tau_{1}^{-1} Z_{0}+\cdots+y^{n-1} \tau_{n-1}^{-1} Z_{0},
$$

where $Z_{0}$ is the maximal order of $Z$, the $\tau_{j}$ are rational integers defined by (29), and $y=(\lambda-u) \beta^{-1}$, with $\beta$ defined by (15) and $\lambda$ varying over the $n$ rational integers defined by (17). Each $M(\lambda)$ contains the order

$$
I=Z_{0}+u Z_{0}+\cdots+u^{n-1} Z_{0}
$$

associated with the cyclic generation $(Z, S, \sigma)$ of $D$.

That $M\left(\lambda_{1}\right)$ is distinct from $M\left(\lambda_{2}\right)$ was proved in $\mathrm{H}, \mathrm{p} .527$, by showing that the corresponding quantities $y=y_{1}, y=y_{2}$ are such that $y_{1}-y_{2}$ is not integral.

5. Maximal orders in direct products. In view of the factorization of any normal division algebra $D$ into a direct product of normal division algebras $D_{i}$ whose degrees are powers of distinct primes, we may inquire whether maximal orders of $D$ can be obtained simply in terms of those of the $D_{i}$. We shall solve this problem under certain hypotheses on the $D_{i}$ and shall obtain some further results on the general problem.

Let $A_{1}$ and $A_{2}$ be cyclic algebras of relatively prime degrees over $R$ and $A=A_{1} \times A_{2}$. A ramification spot of $A$ must be a ramification spot for one of the $A_{i}$. Conversely, suppose that one of the $A_{i}$ does not split at $q$. Then $A_{1 q}$ and $A_{2 q}$ have indices $d_{1}$ and $d_{2}$ which are relatively prime and one of which 
is greater than unity. Hence $A_{q}$ has index $d_{1} d_{2}>1$. Thus the ramification spots of $A$ are those of $A_{1}$ together with those of $A_{2}$.

If $A_{1}$ and $A_{2}$ have cyclic generation fields $Z_{1}$ and $Z_{2}$, respectively, then $A$ has the cyclic generation field $Z_{1} \times Z_{2}$. This fact will be used several times in this section and may be verified by a direct computation and also, for algebras over $R$, in the following way.

Lemma 8. Let $A_{i}=\left(Z_{i}, S_{i}, \sigma_{i}\right)$ be a cyclic algebra of degree $m_{i}$ over $R$, $(i=1,2)$, where $\left(m_{1}, m_{2}\right)=1$. Then $A=A_{1} \times A_{2}$ has a cyclic generation $A=(Z, S, \sigma)$ where $Z=Z_{1} \times Z_{2}, S=S_{1} S_{2}, \sigma=\sigma_{1}{ }^{m_{2}} \sigma_{2}{ }^{m_{1}}$.

The composite of the $Z_{i}$ is their direct product, so that $(Z, S, \sigma)$ has degree $m_{1} m_{2}$ over $R$. If the invariants of $A_{i}$ are denoted by $\nu_{i q}$ for every prime spot $q$ and those of $A$ by $\nu_{q}$, then*

$$
\nu_{q} \equiv m_{2} \nu_{1 q}+m_{1} \nu_{2 q}\left(\bmod m_{1} m_{2}\right) .
$$

We have

$$
\begin{aligned}
(\sigma, Z \mid q) & =\prod_{i, j}\left(\sigma_{i}, Z_{j} \mid q\right)^{m_{1} m_{2} / m_{i}}=\prod_{i}\left(\sigma_{i}, Z_{i} \mid q\right)^{m_{1} m_{2} / m_{i}} \\
& =S_{1}^{{ }^{m_{2} \nu_{1} q}} S_{2}{ }^{m_{1} \nu_{2} q}=\left(S_{1} S_{2}\right)^{m_{2} \nu_{1}+m_{1} \nu_{2} q}=S^{\nu_{q}} .
\end{aligned}
$$

Hence $(Z, S, \sigma)$ has the same invariants $\nu_{q}$ and degree $m_{1} m_{2}$ as $A$. Thus the lemma is proved.

Let $J_{1}$ and $J_{2}$ be any orders in $A_{1}$ and $A_{2}$, respectively, and consider the product $J_{1} J_{2}$ in $A_{1} \times A_{2}$, consisting of all sums of products $a_{1} a_{2}$ with $a_{i}$ in $J_{i}$. The set $J=J_{1} J_{2}$ is an order in $A$ as one can easily verify. If bases of $J_{1}$ and $J_{2}$ over the rational integers are given by $\left(u_{1}, \cdots, u_{m_{1}}{ }^{2}\right)$ and $\left(v_{1}, \cdots, v_{m_{2}}{ }^{2}\right)$, respectively, $J_{1} J_{2}$ has a basis $\left(u_{1} v_{1}, \cdots, u_{i} v_{j}, \cdots, u_{m_{1}}{ }^{2} v_{m_{2}}{ }^{2}\right)$.

Lemma 9. If $J_{i}$ has discriminant $\Delta_{i},(i=1,2)$, then $J=J_{1} J_{2}$ has discriminant

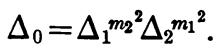

The basis given above for $J$ may be designated by $\left(w_{1}, \cdots, w_{m_{1}{ }^{2} m_{2}}{ }^{2}\right)$, and then $\Delta_{0}=\left|T\left(w_{x} w_{y}\right)\right|$ where $T$ is the trace function in $A$. Let $T_{i}$ be the trace in $A_{i}$, and let $a_{i}$ be any element of $A_{i}$. We shall show that $T\left(a_{1} a_{2}\right)=T_{1}\left(a_{1}\right) T_{2}\left(a_{2}\right)$.

Let $W_{i}$ be a basis of $A_{i}$ relative to a cyclic generation field $Z_{i}$ of $A_{i}$ for $i=1,2$. Then the equation of $a_{i} W_{i}=W_{i} B_{i}$ defines a set of matrices $B_{i}$, with elements in $Z_{i}$, forming an algebra equivalent to $A_{i}$ under the correspondence $a_{i} \longleftrightarrow B_{i}$ for every $a_{i}$ of $A_{i}$, and $T_{i}\left(a_{i}\right)$ is defined to be the trace of the matrix $B_{i}$. Since $m_{1}$ and $m_{2}$ are relatively prime, the composite $Z=Z_{1} \times Z_{2}$ is a cyclic generation field of $A$, and a basis of $A$ relative to $Z$ is given by the vector $W$

* Hasse, Theory of cyclic algebras over an algebraic number field, loc. cit., p. 179, Theorem 4. 
consisting of the products of each of the elements of $W_{1}$ by each of $W_{2}$. Then $a W=W B$ defines a representation $a \longleftrightarrow B$ of $A$, and $T(a)$ is the trace of the matrix $B$. We write $W_{i}=\left(w_{i 1}, \cdots, w_{i m_{i}}\right)$ and have

$$
\begin{aligned}
a_{1} w_{1 r} & =\sum_{f} w_{1 f} b_{1 f r}, \quad a_{2} w_{2 t}=\sum_{\sigma} w_{2 g} b_{2 \sigma t}, \\
a_{1} w_{1 r} a_{2} w_{2 t} & =a_{1} a_{2} w_{1 r} w_{2 t}=\sum_{f, \sigma} w_{1 f} w_{2 g} b_{1 j r} b_{2 g t} .
\end{aligned}
$$

Hence the matrix $B$ corresponding to $a=a_{1} a_{2}$ has elements $b_{1 f r} b_{2 o t}$ and has, as desired, the trace

$$
T\left(a_{1} a_{2}\right)=\sum_{r, t} b_{1 r r} b_{2 t t}=\left(\sum_{r} b_{1 r r}\right)\left(\sum_{t} b_{2 t t}\right)=T_{1}\left(a_{1}\right) T_{2}\left(a_{2}\right) .
$$

Since $w_{x} w_{y}=u_{i} v_{h} u_{j} v_{k}$, we may write $T\left(w_{x} w_{y}\right)=T\left(u_{i} u_{j} v_{h} v_{k}\right)=T_{1}\left(u_{i} u_{j}\right) T_{2}\left(v_{h} v_{k}\right)$. Consider the matrices $C_{1}=\left(T_{1}\left(u_{i} u_{j}\right)\right)$ and $C_{2}=\left(T_{2}\left(v_{h} v_{k}\right)\right)=\left(c_{h k}\right)$. The discriminant $\left|T\left(w_{x} w_{y}\right)\right|$ of $J$ is the determinant $\Delta_{0}=\left|C_{1} c_{h k}\right|$ of a matrix which we have written as a square matrix of $m_{2}{ }^{2}=k_{2}$ rows whose elements are square matrices of $m_{1}{ }^{2}=k_{1}$ rows. When $C_{2}$ is one-rowed, we have $\left|C_{1} c_{k k}\right|=\left|C_{1}\right| k_{2}\left|C_{2}\right|^{k_{1}}$ since then $k_{2}=1$, and we now assume that this formula holds for all matrices $C_{2}$ of $k_{2}-1$ rows. We may assume $c_{11} \neq 0$ and then may replace the blocks $C_{1} c_{h 1}$ by zero matrices under elementary transformations which replace the blocks $C_{1} c_{h k}$ by $C_{1} d_{h k}, d_{h k}=c_{h k}-c_{h 1} c_{1 k} c_{11}{ }^{-1}$. In the remainder of this paragraph the subscripts $h$ and $k$ on $c_{h k}$ will vary over $1, \cdots, k_{2}$ and those on $d_{h k}$ will vary over $2, \cdots, k_{2}$. We have

$$
\Delta_{0}=\left|C_{1} c_{h k}\right|=\left|C_{1} c_{11}\right| \cdot\left|C_{1} d_{h k}\right|=\left|C_{1}\right| \cdot c_{11}^{k_{1}} \cdot\left|C_{1}\right|^{k_{2}-1} \cdot\left|d_{h k}\right|^{k_{1}}
$$

by our induction. But $\left|c_{h k}\right|=c_{11}\left|d_{h k}\right|$ so that $\Delta_{0}=\left|C_{1}\right|^{k_{2}} \cdot\left|C_{2}\right|^{k_{1}}$, and the lemma is proved.

The discriminant of $A$ is the product*

$$
\Delta=\prod_{q} q^{e_{q}}, \quad e_{q}=\left(n_{q}-1\right) n^{2} / n_{q},
$$

where $g$ varies over all ramification spots of $A, n$ is the degree $m_{1} m_{2}$ of $A$, and $n_{q}$ is the $q$-index of $A$. Then $n_{q}=m_{1 q} m_{2 q}$ where $m_{i q}$ is the $q$-index of $A_{i}$. Let $\Delta_{i}$ be the discriminant of $A_{i}$. Then a direct computation shows that if $A_{1}$ and $A_{2}$ have no ramification spots in common, the discriminant of $A$ is

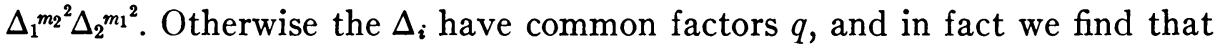
in general $A$ has discriminant

$$
\Delta=\Delta_{1}^{m^{2} \Delta_{2}{ }^{2}{ }^{2}} \prod_{q} q^{-\left(m_{1 q}-1\right)\left(m_{2 q}-1\right) n^{2} / n_{q}}
$$

* Reichhardt, op. cit. 
where the product is taken over all common ramification spots $q$ of $A_{1}$ and $A_{2}$. An immediate consequence of this formula and Lemma 9 is stated now.

THEOREM 4. Let $A_{1}$ and $A_{2}$ be normal simple algebras of relatively prime degrees $m_{1}$ and $m_{2}$ over $R$, and let $M_{1}$ and $M_{2}$ be any maximal orders in $A_{1}$ and $A_{2}$, respectively. Then $M_{1} M_{2}$ is a maximal order in $A=A_{1} \times A_{2}$ if and only if the discriminants $\Delta_{1}$ and $\Delta_{2}$ of $A_{1}$ and $A_{2}$ are relatively prime. In this case the discriminant of. $A$ is $\Delta_{1}^{m_{2}{ }^{2}} \Delta_{2}^{m_{1}{ }^{2}}$.

This is an analogue of a known theorem* on algebraic fields over $R$ with relatively prime discriminants. That $M=M_{1} M_{2}$ is maximal may also be proved by using Hasse's determination $\dagger$ of all maximal orders in the $q$-adic algebra $A_{q}$. We show by this means that for every prime spot $q$ the $q$-component $M_{q}$ is a maximal order of $A_{q}$. But this is a necessary and sufficient condition that $M$ be maximal in $A$.

An application of Lemma 8 and Theorem 1 yields the following result which may be useful in the determination of maximal orders in a direct product.

Theorem 5. Let $D$ be a direct product $D_{1} \times \cdots \times D_{t}$ of normal division algebras $D_{i}$ of Theorem 1 such that the degrees $m_{i}$ of the $D_{i}$ are relatively prime in pairs, and let $n=m_{1} \cdots m_{t}$. Then each $D_{i}$ has a cyclic generation $\left(Z_{i}, S_{i}, \sigma_{i}\right)$ as described in Theorem 1 , and $D$ has a cyclic generation

$$
D=(Z, S, \sigma), \quad Z=Z_{1} \times \cdots \times Z_{t}, \quad S=S_{1} \cdots S_{t}, \quad \sigma=\prod_{i} \sigma_{i}^{n / m_{i}} .
$$

The generations of the $D_{i}$ may be chosen so that the conductors $p_{1}, \cdots, p_{t}$ of $Z_{1}, \cdots, Z_{t}$ are distinct primes, and are not ramification spots of $D$. The former property implies that the maximal order $Z_{0}$ of $Z$ is the product of the maximal orders $Z_{0 i}$ of the fields $Z_{\imath}$.

* D. Hilbert, Gesammelte Abhandlungen, vol. 1, 1932, p. 146. The result of Theorem 4 was also obtained in a different way by K. Shoda and T. Nakamura in the paper Über das Produkt zweier Algebrenklassen mit zueinander primen Diskriminanten, Proceedings of the Imperial Academy of Japan, vol. 10 (1934), pp. 443-446.

$\dagger \mathrm{H}$. Hasse, Über p-adische Schiefkörper und ihre Bedeutung für die Arithmetik hyperkomplexer Zahlsysteme, Mathematische Annalen, vol. 104 (1931), pp. 495-534, Theorem 47.

The University of Chicago,

Chicago, Ill. 